\title{
Preoperative risk stratification for early recurrence of HBV-related hepatocellular carcinoma after deceased donor liver transplantation: a five-eight model development and validation
}

\author{
Abdulahad Abdulrab Mohammed Al-Ameri ${ }^{1,2}$, Xuyong Wei ${ }^{1,2}$, Lidan Lin ${ }^{3}$, Zhou Shao ${ }^{1,2}$, Haijun Guo ${ }^{1,2}$, \\ Haiyang Xie ${ }^{1,2}$, Lin Zhou ${ }^{1,2}$, Shusen Zheng ${ }^{1,2,3}$ and Xiao Xu u, $^{1,3^{*}}$
}

\begin{abstract}
Background: Early recurrence of hepatocellular carcinoma (HCC) after liver transplantation (LT) is associated with poor surgical outcomes. This study aims to construct a preoperative model to predict individual risk of post-LT HCC recurrence.

Methods: Data of 748 adult patients who underwent deceased donor LT for HCC between January 2015, and February 2019 were collected retrospectively from the China Liver Transplant Registry database and randomly divided into training $(n=486)$ and validation $(n=262)$ cohorts. A multivariate analysis was performed and the fiveeight model was developed.
\end{abstract}

Results: A total of 748 patients were included in the study; of them, 96\% had hepatitis B virus (HBV) and 84\% had cirrhosis. Pre-LT serum alpha-fetoprotein (AFP), tumor number and largest tumor diameter were incorporated to construct the 5-8 model which can stratify patients accurately according to their risk of recurrence into three prognostic subgroups; low-(0-5 points), medium-(6-8 points) and high-risk (> 8 points) with 2-year post-LT recurrence rate of $(5,20$ and $51 \%, p<0.001)$ respectively. The $5-8$ model was better than Milan, Hangzhou, and AFPmodel for prediction of HCC early recurrence. These findings were confirmed by the results of the validation cohort.

Conclusions: The 5-8 model is a simple validated and accurate tool for preoperative stratification of early recurrence of HCC after LT.

Keywords: Liver transplantation, Hepatoma, Milan criteria, Hangzhou criteria,prognosis,relapse

\section{Background}

Globally, hepatocellular carcinoma (HCC) is the sixth most common malignancy and the third leading cause of cancer-related deaths [1]. In the Pacific region, China accounted for $84.6 \%$ of $\mathrm{HCC}$ incidence and $86.3 \%$ of

\footnotetext{
* Correspondence: zjxu@zju.edu.cn

${ }^{1}$ Department of Hepatobiliary and Pancreatic Surgery, First Affiliated Hospital, Zhejiang University School of Medicine; Institution of Organ Transplantation, Zhejiang University, Hangzhou 310003, China

${ }^{2}$ NHFPC Key Laboratory of Combined Multi-organ Transplantation, Hangzhou 310003, Zhejiang Province, China

Full list of author information is available at the end of the article
}

HCC mortality with Hepatitis B virus (HBV) infection as the most common cause [2].

Although liver transplantation (LT) is an excellent therapeutic choice for HCC as the patients who received LT have the highest chance of cure among all other therapies, the organs shortage is still a main challenge [3]. For this, several selection criteria of HCC candidates for LT were proposed, of them, Milan and Hangzhou criteria are the most recommended tools by the Chinese Society of Organ Transplantation $[4,5]$. The high rates of $\mathrm{HCC}$ recurrence after LT which have been reported

(c) The Author(s). 2019 Open Access This article is distributed under the terms of the Creative Commons Attribution 4.0 International License (http://creativecommons.org/licenses/by/4.0/), which permits unrestricted use, distribution, and reproduction in any medium, provided you give appropriate credit to the original author(s) and the source, provide a link to the Creative Commons license, and indicate if changes were made. The Creative Commons Public Domain Dedication waiver (http://creativecommons.org/publicdomain/zero/1.0/) applies to the data made available in this article, unless otherwise stated. 
to be $8-30 \%[3,6]$ remains an important cause of death for HCC patients.

Alpha-fetoprotein (AFP) is a potential biomarker for early diagnosis and prediction of HCC recurrence. High level of preoperative AFP, which can be seen in approximately $60 \%$ of the HCC patients, is a risk factor for $\mathrm{HCC}$ recurrence and can be used to define at-risk HCC patients $[7,8]$. In addition to AFP, there are well recognized preoperative risk factors which reflect the biological behavior of HCC and closely associated with post-LT HCC recurrence including vitamin $\mathrm{K}$ absence-II and neutrophil-to-lymphocyte ratio $[9,10]$. The predominant morphological factors that show correlation with higher rates of HCC recurrence after LT include; tumor number and size $[11,12]$.

These risk factors were employed in a collective fashion to establish different predictive models for HCC recurrence, however unavailability of an effective, validated and reliable model to stratify patients preoperatively of HCC recurrence makes a unified practice across different countries out of reach. So, careful preoperative risk stratification of $\mathrm{HCC}$ recurrence is not only crucial for better management, but also very helpful to define a risk-based prioritizing strategy for selection of HCC candidates for LT.

In this multicenter study, we established a preoperative predictive model for early recurrence of HCC after LT. This model could be used as an adjuvant tool beside the conventional selection criteria to predict postoperative prognosis at a personal level more accurately.

\section{Methods}

The design of this study followed the Transparent Reporting of a multivariable prediction model for Individual Prognosis or Diagnosis (TRIPOD) Statement [13]. This study has been approved by the Scientific Committee of the China Liver Transplant Registry (http://www. cltr.org) which is in accordance with ethical guidelines of Helsinki Declaration 1975, as revised in 2013. Written informed consent was obtained. Data of 1512 consecutive patients who underwent LT were retrospectively recalled from the prospectively maintained database of (CLTR) from 2015 January to 2019 February. Inclusion criteria were [1] adult patients with age $\geq 18$ [2] preoperative radiologically diagnosed HCC depending on guidelines of the current guidelines of American Association for the Study of Liver Diseases (AASLD) [14] [3] no history of previous LT or combined hepatorenal transplantation [4] patients who survived at least 3 months after the date of surgery [5] no incidental HCC [6] all the clinical and laboratory data required for the analysis are available. After applying the inclusion criteria, 748 patients were involved in the final analysis and divided randomly into training $(n=486)$ and validation
( $n=262)$ cohorts. Data collection were performed by independent researchers blinded to statistical analysis. The collected clinicopathological variables included; age, gender, diabetes and hypertension, body mass index (BMI), presence of hepatitis B virus (HBV) infection, cirrhosis, Model for End-stage Liver Disease (MELD), Child score, neoadjuvant therapy (i.e. transarterial chemoembolization (TACE), radiofrequency ablation (RFA) and hepatectomy), donor type, donor death cause. The Pre-LT characteristics of $\mathrm{HCC}$ were obtained from radiological assessment (mainly CT, MRI), including the total tumor diameter, largest tumor diameter, number of nodules and the last pre-LT measurements of AFP. Post-LT features of HCC were obtained from the pathology reports including lymphovascular invasion and tumor differentiation according to the modified Edmondson score [15]. Data of survival and recurrence, including death cause, last follow-up dates, recurrence and death dates. Milan, Hangzhou criteria and AFP model were calculated $[4,5,16]$. The last censoring date of this study was 21st February 2019.

\section{Outcome and definitions}

The primary outcome of this study is 2-year recurrence rate of HCC after LT. The recurrence was considered as 'early' if the time from LT to recurrence was $\leq 2$ years [17]. Time to recurrence was calculated from the date of LT surgery to the date of recurrence diagnosis or last follow-up. The corresponding overall survival (OS) was also calculated from the date of LT surgery to date of death or last follow-up.

The selection criteria for LT included in this study are Milan and Hangzhou criteria. The former required the absence of distance metastasis and macrovascular invasion and included patients with a single nodule $\leq 5 \mathrm{~cm}$ or $\leq 3$ nodules (each nodule $\leq 3 \mathrm{~cm}$ ) [4], while the latter required the absence of macrovascular invasion and included patients with (a) total tumor size $\leq 8 \mathrm{~cm}$, (b) total tumor diameter $>8 \mathrm{~cm}$, well moderate tumor differentiation and preoperative AFP level $\leq 400 \mathrm{ng} / \mathrm{mL}$, concurrently [5].

AFP model is a binary tool incorporated largest tumor size, number of nodules and pre-LT AFP (at 100 and 1000 $\mathrm{ng} / \mathrm{mL}$ ) with a cut-off value of two points to discriminate patients within and outside the AFP model patients [16].

The death was defined as HCC- related death if there is an evidence of HCC recurrence post-LT or documented metastasis and/or vascular invasion otherwise it was considered as HCC-unrelated death. The last censoring date or the date of events (recurrence and death) were considered after following up all patients.

\section{Postoperative management and follow up}

Generally, postoperative immunosuppressants consisted of calcineurin inhibitors and steroids. Steroids were 
withdrawn within 3 months. Follow up of the patients were done every 3-6 months during the first 2 years post-LT. During follow up time, in addition to AFP measurement, abdominal computed tomography (CT) scan and magnetic resonance imaging (MRI) were performed according to their indications.

\section{Statistical analysis}

Statistical analysis was performed using Stata MP 14. Categorical data were reported as values and percentages and compared using Fisher's exact test or Chi-Square test. Continuous data were reported as mean $\pm \mathrm{SD}$ or median (interquartile range [IQR]) and compared with Student's T-test or rank sum test according to their distribution respectively. Recurrence and survival probabilities were estimated by Kaplan-Meier (KM) methods and compared using the log-rank test (Mantel-Cox). Univariable and multivariable Cox regression analyses for factors affecting post-LT HCC recurrence were performed by Cox proportional hazards regression models. Variables after a univariable analysis with a $P$-value $<0.05$ were included in the multivariable analysis, and the final model was constructed by stepwise backward selection (Wald test). Notebaly,the potential cut-off values were estimated in accordance with previous studies in the literature and using the Akaike information criterion (AIC), the cut-off points with the lowest AIC values were selected to be included in the final model. Proportional-hazards assumption was assessed by the Schoenfeld test and by visual assessment of log-log survival curves. The training and validation cohorts were compared. The discriminatory performance of 5-8 Model, Milan, Hangzhou, and AFP model was calculated and compared using Harrell's C and Somers' D statistics [18] and also was assessed visually via KM curves. Moreover, the competing risk analysis was also performed to evaluate the cumulative incidence of HCC related and unrelated deaths [19]. A two-tailed $p$-value of $<0.05$ indicates a statistically significance difference.

\section{Results}

A total of 748 patients were included in the study with a mean age of $51.6 \pm 8.6$ and $89.6 \%$ were male. HBV infection was the most common cause (96\%) and cirrhosis was found in $84 \%$. The 486 patients of the training cohort had similar characteristics to the 262 patients of validation cohort without any significant differences as summarized in (Table 1). For the training and validation cohorts, the median post-LT follow-up was 338 days, (IQR: 205-673 days) and 416.5 days, (IQR:205-672 days), respectively. The 2-year OS was $82.6 \%$ (95\%CI:0.770.87 ) vs $81.9 \%$ (95\% CI: $0.74-0.87$ ), $p=0.870$ (Fig. 1a). Recurrence of HCC was observed in $12.8 \%$ (62 of 486) vs $16.8 \%$ (44 of 262 ) at a median of 11.3 months (IQR:
5.9-21.2, months) vs 12.0 months IQR:6.2-20.5 months) after LT. The 2-year overall HCC recurrence was $17.1 \%$ (95\% CI:0.13-0.22) vs $25.9 \%$ (95\% CI: 0.19-0.36), $p=0.180$ (Fig. 1b).

\section{Factors affecting post-LT HCC recurrence}

Factors of post-LT HCC recurrence in the training cohort were identified by univariable Cox regression analysis (Table 2). The preoperative factors that associated with post-LT HCC recurrence included pre-LT TACE, pre-LT AFP, total tumor diameter $(\mathrm{cm})$, the largest tumor diameter $(\mathrm{cm})$ and the number of nodules at all tested cut-off values. The postoperative factors included; vascular invasion, poorly differentiated tumor grade. Other factors including recipient age, gender, MELD score, number of HCC nodules, use of other neoadjuvant therapies (i.e. hepatectomy, RFA) did not associate with post-LT HCC recurrence. We emphasize that the tumor diameters and the number of nodules were obtained from the last imaging before transplantation by which Milan and Hangzhou criteria were calculated. On multivariable analysis, only pre-LT AFP, largest tumor size and tumor number were the preoperative predictors found to be associated with increasing the risk of postLT HCC recurrence. While the postoperative predictors were the only presence of vascular invasion and poorly differentiated tumor grade.

\section{Development of the 5-8 score}

Preoperative factors with $p$-value $<0.05$ on univariable analysis were then used in the multivariable model. A Cox regression analysis was then performed with backward selection to conduct a multivariable analysis of clinicopathologic factors that associated significantly with post-LT HCC recurrence. Preoperative independent factors of post-LT HCC recurrence were utilized to construct the 5-8 score including (1) pre-LT AFP (at the following cut-off values: $10-200,201-1000$, and $>1000$ $\mathrm{ng} / \mathrm{mL}$ ), (2) the largest diameter of tumor (at the following cut-off values: $4-6,6.1-8$ and $>8$ ), and (3) number of nodules (single vs multiple). It is important to note that the model with the lowest (AIC), was chosen as the final model for the risk score. The multivariable HR of these factors derived from the Cox regression model were rounded to the nearest integer, then used to calculate the simplified 5-8 score. To calculate the score for each patient, the individual points for each of the three variables can be added together giving a minimum point of 0 and a maximum point of 24(Table 3).

\section{Prediction of $\mathrm{HCC}$ recurrence risk by the 5-8 score}

Based on the 5-8 score, patients were then accurately stratified according to their risk of recurrence into three categories; the low-risk group had a score of 0 to 5 , the 
Table 1 Baseline characteristics of training and validation cohorts

\begin{tabular}{|c|c|c|c|}
\hline Variable & Training cohort $(n=486)$ & Validation cohort $(\mathrm{n}=262)$ & $P$-value \\
\hline$\overline{\text { Age }(\text { years })^{a}}$ & $51.6 \pm 8.6$ & $51.5 \pm 8.7$ & 0.890 \\
\hline Gender (Male/Female) $^{\text {b }}$ & $439(90.3) / 47(9.7)$ & $231(88.1) / 31(11.8)$ & 0.356 \\
\hline Diabetes (Yes/No) ${ }^{b}$ & $62(12.8) / 424(87.2)$ & $40(15.3) / 222(84.7)$ & 0.340 \\
\hline Hypertension (Yes/No) ${ }^{b}$ & $48(9.9) / 438(90.1)$ & $33(12.6) / 229(87.4)$ & 0.254 \\
\hline $\mathrm{BMI}^{\mathrm{a}}$ & $24.4 \pm 12.9$ & $23.3 \pm 3.8$ & 0.162 \\
\hline Cause $(\mathrm{HBV} / \mathrm{Non}-\mathrm{HBV})^{\mathrm{b}}$ & $462(95.1) / 24(4.9)$ & $256(97.7) / 6(2.3)$ & 0.078 \\
\hline Cirrhosis (Yes/No) ${ }^{b}$ & $411(84.6) / 75(15.4)$ & $217(82.8) / 45(17.2)$ & 0.535 \\
\hline \multicolumn{4}{|l|}{ Pre-LT AFP $(n g / m L){ }^{b}$} \\
\hline$\leq 10$ & $164(33.7)$ & $94(35.9)$ & \multirow[t]{4}{*}{0.621} \\
\hline $10-200$ & $167(34.4)$ & $95(36.3)$ & \\
\hline $200-1000$ & $82(16.9)$ & $35(13.4)$ & \\
\hline$>1000$ & $73(15.0)$ & $38(14.5)$ & \\
\hline \multicolumn{4}{|l|}{ Total tumor diameter $(\mathrm{cm})^{\mathrm{b}}$} \\
\hline$\leq 5$ & $283(58.2)$ & $148(56.5)$ & \multirow[t]{3}{*}{0.880} \\
\hline $5.1-8$ & $104(21.4)$ & $57(21.8)$ & \\
\hline$>8$ & $99(20.4)$ & $57(21.8)$ & \\
\hline \multicolumn{4}{|l|}{ Largest tumor diameter $(\mathrm{cm})^{\mathrm{b}}$} \\
\hline$\leq 4$ & 309 (63.6) & $162(61.8)$ & \multirow[t]{4}{*}{0.296} \\
\hline $4-6$ & $83(17.1)$ & $56(21.4)$ & \\
\hline $6.1-8$ & $42(8.6)$ & $15(5.7)$ & \\
\hline$>8$ & $52(10.7)$ & $29(11.1)$ & \\
\hline Tumor number (single/multiple) ${ }^{b}$ & $296(60.9) / 190(39.1)$ & $151(57.6) / 111(42.3)$ & 0.384 \\
\hline MELD ${ }^{c}$ & $12[8-21],(6-51)$ & 11 [9-19], (6-44) & 0.503 \\
\hline Child score $^{c}$ & 7 [5-9], (5-14) & $7[5-9],(5-14)$ & 0.473 \\
\hline \multicolumn{4}{|l|}{ Neoadjuvant therapy ${ }^{\text {b }}$} \\
\hline TACE (yes/no) & $195(40.1) / 291(59.9)$ & $113(43.1) / 149(56.9)$ & 0.425 \\
\hline RFA (yes/no) & $85(17.5) / 401(82.5)$ & $49(18.7) / 213(81.3)$ & 0.680 \\
\hline Hepatectomy (yes/no) & $83(17.1) / 403(82.9)$ & $41(15.7) / 221(84.4)$ & 0.616 \\
\hline Donor type ${ }^{b}$ DBD/DCD/DBCD & $142(29.2) / 174(35.8) / 170(35.0)$ & $70(26.7) / 103(39.3) / 89(34.0)$ & 0.609 \\
\hline \multicolumn{4}{|l|}{ Donor death cause ${ }^{b}$} \\
\hline Trauma & $233(47.9)$ & $138(52.7)$ & \multirow[t]{5}{*}{0.404} \\
\hline CVA & $190(39.0)$ & $94(35.9)$ & \\
\hline Tumor & $29(6.0)$ & $10(3.8)$ & \\
\hline Anoxia & $17(3.5)$ & $13(4.9)$ & \\
\hline Others & $17(3.5)$ & $7(2.7)$ & \\
\hline \multicolumn{4}{|l|}{ Differentiation ${ }^{b}$} \\
\hline well & $72(14.8)$ & $42(16.0)$ & \multirow[t]{3}{*}{0.884} \\
\hline moderate & $338(69.6)$ & $178(67.9)$ & \\
\hline poor & $76(15.6)$ & $42(16.0)$ & \\
\hline Vascular invasion (yes/no) ${ }^{b}$ & $113(23.3) / 373(76.8)$ & $74(28.2) / 188(71.8)$ & 0.132 \\
\hline Milan (in/out) ${ }^{b}$ & $259(53.3) / 227(46.7)$ & $125(47.7) / 137(52.3)$ & 0.145 \\
\hline Hangzhou (in/out) ${ }^{b}$ & $390(80.3) / 96(19.8)$ & $197(75.2) / 65(24.8)$ & 0.108 \\
\hline AFP model (in/out) ${ }^{b}$ & $301(61.9) / 185(38.1)$ & $167(63.7) / 95(36.3)$ & 0.626 \\
\hline Post-LT mortality (Died/alive) ${ }^{\text {b }}$ & $55(11.3) / 431(88.7)$ & $31(11.8) / 231(88.2)$ & 0.833 \\
\hline
\end{tabular}


Table 1 Baseline characteristics of training and validation cohorts (Continued)

\begin{tabular}{llll}
\hline Variable & Training cohort $(\mathrm{n}=486)$ & Validation cohort $(\mathrm{n}=262)$ & $P$-value \\
\hline Post-LT recurrence $(\mathrm{yes} / \mathrm{no})^{\mathrm{b}}$ & $62(12.8) / 424(87.2)$ & $44(16.8) / 218(83.2)$ & 0.131 \\
Time to recurrence $\left(\right.$ months) $^{c}$ & $11.3[5.9-21.2],(0.2-47.0)$ & $12.0[6.2-20.5],(1.1-44.8)$ & 0.863 \\
Follow-up (days) & $388[205-673],(92-1428)$ & $416.5[205-672],(92-1363)$ & 0.802
\end{tabular}

Note: BMI Body mass index, HBV Hepatitis B virus infection, AFP Alpha-fetoprotein, MELD Model for End-Stage Liver Disease, $L T$ Liver transplantation, TACE Transarterial chemoembolization, RFA Radiofrequency ablation, $D B D$ Donation after brain death, $D C D$ Donation after circulatory death, $D B C D$ Donation after brain death followed by circulatory death, CVA Cerebrovascular accident, ${ }^{a}$ Mean $\pm \mathrm{SD},{ }^{\mathrm{b}}$ number (percentage), ${ }^{\mathrm{c}}$ (median, [IQR, interquartile range]),(range)

medium-risk group had a score of 6-8, the high-risk group had a score of $>8$ (Table 3 ). The most common group being low risk group $[n=253(52.1 \%)]$ then medium-risk group $[n=129(26.5 \%)]$ and high-risk group $(n=104(21.4 \%)]$. The risk of 2-year HCC recurrence was increased significantly from low to high-risk group as shown by KM curves and log-rank test. The 2-year HCC recurrence rate was $4.5 \%$ (95\% CI:0.02-0.09), $20.0 \%$ (95\% CI:0.12-0.34) and 51.4\% (95\% CI:0.36-0.73) in the low, medium and high-risk group respectively (overall log-rank $p<0.001)$ )(Fig. 2a). The corresponding 2- year OS was $92.5 \%$ (95\% CI: 0.86-0.96), 82.9\%(95\% CI:0.720.90 ) and $57.2 \%$ (95\% CI: $0.42-0.70$ ), respectively (overall $\log$-rank $p<0.001$ ) (Fig. 2b). According to the 5-8 score, patients before transplantation with AFP level of $\leq 10 \mathrm{ng} /$ $\mathrm{mL}$ and single nodule with the largest tumor diameter of $<4 \mathrm{~cm}$ on radiological assessment would be categorized in the low-risk group, in contrast to patients who have pre-LT AFP of $>1000(\mathrm{ng} / \mathrm{mL})$ and largest tumor diameter of $>8 \mathrm{~cm}$, will be categorized in high-risk category. There was no deviation from the proportional hazard assumption according to the visual inspection of $\log$-log survival curves and the Schoenfeld test $(p=0.708)$. Also, for prediction HCC recurrence in the training cohort, visual assessment of the KM curves showed good discrimination between the three 5-8 model prognostic subgroups. Moreover, the Harrell's C and Somers' D of 5-8score were $79 \%(95 \% \mathrm{CI}: 0.73-0.86)$ and 59\%(95\% CI: 0.40-0.72) (Table 4). Based on the competing risk analysis, the 2-year cumulative incidence of mortality, while controlling for the risk of HCC-related death, was 5.2\%(95\% CI: $0.02-0.11), 12.9 \%(95 \%$ CI: $0.06-0.22)$ and $35.0 \%$ (95\% CI: $0.22-0.49$, overall $p<0.001$ ) in patients with low, medium and high 5-8 score (Fig. 2c). Furthermore, 2- year cumulative incidence of $\mathrm{HCC}$-unrelated death which not related to HCC recurrence were, 2.4\%(95\% CI: $0.01-0.05), 4.2 \%$ (95\% CI: $0.01-0.10)$ and 7.9\%(95\% CI: $0.03-0.16$ ), (overall $p=0.120$ ) in patients with low, medium and high 5-8score (Fig. 2d).

\section{Comparison of the 5-8 model with Milan and Hangzhou criteria}

The 2-years recurrence rate for patients meeting and exceeding Milan criteria, was 4.8 and $33.8 \%$ respectively $(p<0.001)$ (Fig. 3a), while it was $9.1 \%$ vs $57.5 \%$ in patients meeting and exceeding Hangzhou criteria respectively, $(p<0.001)$ (Fig. 3d). We further analyzed the riskstratified patients of 5-8 score for the patients who were fulfilling and exceeding either Milan or Hangzhou criteria. Among 259 patients who were fulfilling Milan criteria (53.2\%), the risk of 2-year HCC recurrence according to $5-8$ score was $2.6 \%$ (95\% CI: $0.01-0.07$ ) in the low risk group, $8.5 \%$ (95\% CI: $0.03-0.29$ ) in mediumrisk group and $23.6 \%$ (95\%CI: $0.06-0.95$ ) in the high-risk
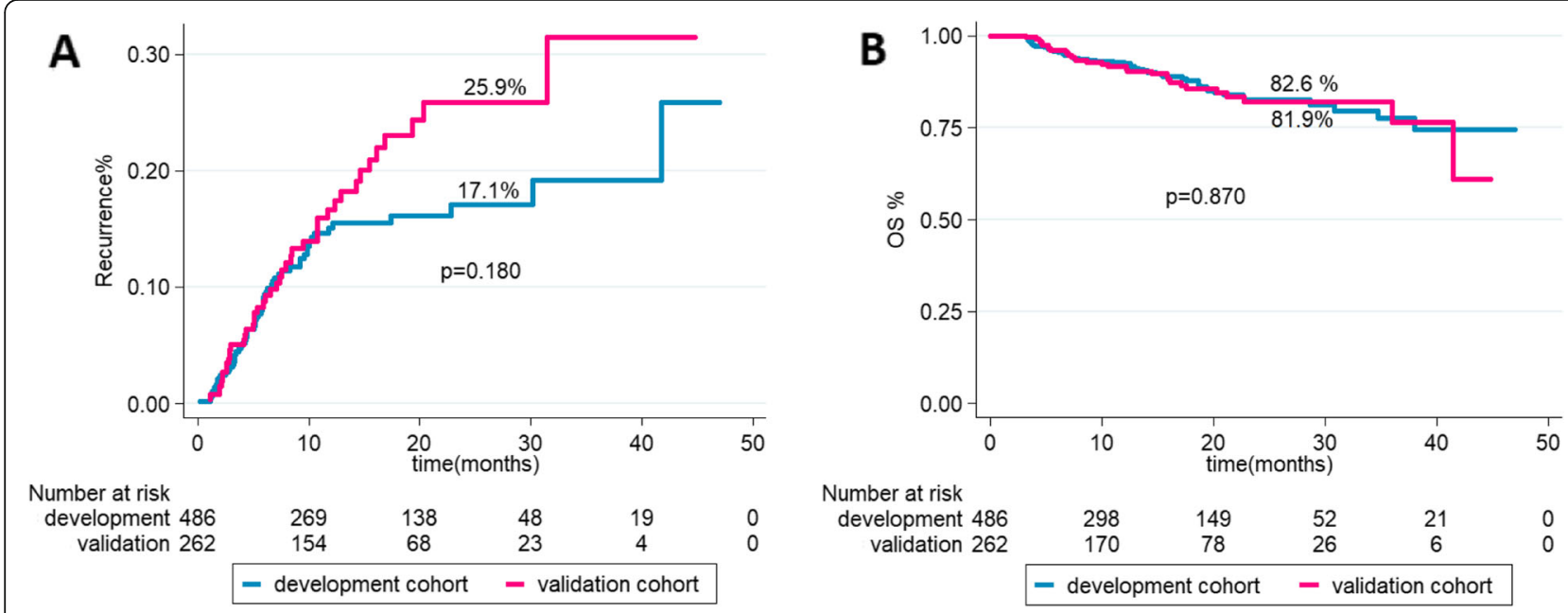

Fig. 1 The 2-year recurrence and overall survival rates in the training (a) and validation(b) cohorts 
Table 2 Univariable cox analysis of risk factors for early recurrence of HCC

\begin{tabular}{|c|c|c|c|c|c|c|}
\hline Variable & 2-year recurrence rate & $\mathrm{HR}$ & SE & $z$ & $P>z$ & $95 \% \mathrm{Cl}$ \\
\hline 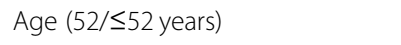 & $17.6 / 16.4$ & 0.98 & 0.25 & 0.07 & 0.942 & $0.59-1.63$ \\
\hline Gender (male/female) & $17.9 / 9.2$ & 1.51 & 0.78 & 0.80 & 0.426 & $0.55-4.16$ \\
\hline Diabetes (yes/no) & 19.4/17.1 & 0.79 & 0.34 & 0.55 & 0.585 & $0.34-1.84$ \\
\hline Hypertension (yes/no) & $24.7 / 16.3$ & 1.54 & 0.59 & 1.14 & 0.253 & $0.73-3.25$ \\
\hline BMI $(25 / \leq 25)$ & $18.5 / 16.4$ & 0.99 & 0.28 & 0.05 & 0.959 & 0.561 .73 \\
\hline CTP class(A/B/C) & 19.5/14.9/15.3 & 0.94 & 0.16 & 0.38 & 0.705 & $0.67-1.31$ \\
\hline $\operatorname{MELD}(12 / \leq 12)$ & $13.7 / 20.2$ & 0.81 & 0.21 & 0.81 & 0.416 & $0.49-1.35$ \\
\hline Cirrhosis (yes/no) & 17.414 .2 & 1.24 & 0.47 & 0.57 & 0.566 & $0.59-2.62$ \\
\hline \multicolumn{7}{|l|}{ Pre-LT AFP (ng/mL) (Reference, $\leq 10)$} \\
\hline $10-200$ & 19.5 & 3.57 & 1.65 & 2.75 & 0.006 & $1.44-8.83$ \\
\hline $201-1000$ & 21.8 & 4.65 & 2.29 & 3.11 & 0.002 & $1.77-12.23$ \\
\hline$>1000$ & 39.2 & 9.03 & 4.20 & 4.72 & $<0.001$ & $3.62-22.49$ \\
\hline \multicolumn{7}{|c|}{ Total tumor diameter $(\mathrm{cm})$ (Reference, $\leq 5$ ) } \\
\hline $5-8$ & 21.9 & 3.20 & 1.19 & 3.13 & 0.002 & $1.55-6.64$ \\
\hline$>8$ & 52.6 & 8.89 & 2.88 & 6.75 & $<0.001$ & 4.7116 .76 \\
\hline \multicolumn{7}{|c|}{ Largest tumor diameter $(\mathrm{cm})$ (Reference, $\leq 4$ ) } \\
\hline $4-6$ & 21.8 & 2.37 & 0.90 & 2.28 & 0.023 & $1.13-4.98$ \\
\hline $6-8$ & 28.2 & 3.67 & 1.55 & 3.08 & 0.002 & $1.61-8.39$ \\
\hline$>8$ & 74.8 & 10.49 & 3.31 & 7.45 & $<0.001$ & $5.65-19.46$ \\
\hline Nodules number (single/multiple) & $12.3 / 24.6$ & 1.883142 & .4874233 & 2.45 & 0.014 & $1.13-3.13$ \\
\hline Pre-LT TACE (yes/no) & 23.3/13.0 & 2.03 & 0.53 & 2.73 & 0.006 & $1.22-3.39$ \\
\hline Pre-LT RFA (yes/no) & 13.6/18.0 & 0.79 & 0.29 & 0.65 & 0.519 & $0.39-1.61$ \\
\hline Pre-LT hepatectomy (yes/no) & 25.0/15.5 & 1.59 & 0.49 & 1.52 & 0.128 & $0.88-2.90$ \\
\hline Vascular invasion (yes/no) & 43.0/9.8 & 4.31 & 1.12 & 5.62 & $<0.001$ & $2.59-7.17$ \\
\hline \multicolumn{7}{|l|}{ Differentiation (Reference, well) } \\
\hline moderate & 14.9 & 1.52 & 0.67 & 0.95 & 0.344 & $0.64-3.59$ \\
\hline poor & 26.1 & 2.81 & 1.35 & 2.16 & 0.031 & $1.10-7.19$ \\
\hline Milan criteria (out/in) & $33.8 / 4.8$ & 7.68 & 2.78 & 5.64 & $<0.001$ & $3.78-15.62$ \\
\hline Hangzhou criteria (out/in) & $57.5 / 9.1$ & 7.24 & 1.91 & 7.52 & $<0.001$ & $4.32-12.13$ \\
\hline AFP model (out/in) & $35.0 / 7.9$ & 5.80 & 1.73 & 5.89 & $<0.001$ & $3.23-10.41$ \\
\hline
\end{tabular}

Note: BMI Body mass index, CTP Child-Turcotte-Pugh, AFP Alpha-fetoprotein, MELD Model for End-Stage Liver Disease, LT Liver transplantation, TACE Transarterial chemoembolization, RFA Radiofrequency ablation

group (overall $p=0.009$ ) (Fig. 3b). While for 227 patients who are exceeding Milan criteria (46.7\%), the risk of 2year HCC recurrence was $11.5 \%$ (95\% CI: 0.05-0.28), 29.0\% (95\% CI:0.16-0.51) and 55.0\%(95\% CI: 0.38-0.79), respectively (overall $p<0.001$ ) (Fig. 3c). Likewise, for the 390 patients within Hangzhou criteria (80.3\%), the risk of 2-year HCC recurrence according to 5-8 score was $3.0 \%$ (95\% CI:0.01-0.07) in the low risk group, $16.3 \%$ (95\% CI:0.09-0.31) in medium- risk group and $24.6 \%$ (95\% CI: $0.12-0.49)$ in the high-risk group (overall $p<$ 0.009) (Fig. 3e). While for 96 patients who are exceeding Milan criteria (19.8\%), the risk of 2-year HCC recurrence was $26.2 \%$ (95\% CI: $0.08-0.82), 37.2 \%(95 \%$ CI: $0.16-$ 0.87 ) and $79.3 \%$ (95\% CI:0.52-1.21), respectively (overall $p<0.001$ ) (Fig. 3f). For prediction of HCC recurrence, Harrell's C and Somers' D of 5-8score were 79\%(95\% CI: $0.73-0.86)$ and $59 \%(95 \%$ CI:0.40-0.72) in the training cohort compared with $72 \%(95 \%$ CI:0.67-0.76) and 43\%(95\% CI:0.35-0.58) for Milan criteria and 72\%(95\% CI:0.65-0.78) and 43\%(95\% CI:0.31-0.61) for Hangzhou criteria (Table 4).

\section{Comparison of the 5-8 model with AFP model}

For patients exceeding and fulfilling the AFP model, the 2-year rates of HCC recurrence were 35.0 and $7.9 \%$, respectively (Fig. 4a). Further analysis for the risk-stratified patients of 5-8 score for the patients who were within and outside the AFP model showed that among 301 
Table 3 Multivariate Cox analysis of risk factors for early recurrence of HCC

\begin{tabular}{|c|c|c|c|c|c|c|c|}
\hline Variable & $H R$ & SE & $z$ & $P>z$ & $95 \% \mathrm{Cl}$ & $\beta$ Coefficient & Points \\
\hline \multicolumn{8}{|c|}{ Pre-LT AFP (ng/mL) } \\
\hline$\leq 10$ & 1 & - & - & - & - & - & 0 \\
\hline 10-200 & 3.12 & 1.51 & 2.29 & 0.022 & $1.18-8.25$ & 1.29 & 3 \\
\hline $200-1000$ & 3.62 & 1.69 & 2.77 & 0.006 & $1.46-9.05$ & 1.14 & 4 \\
\hline$>1000$ & 6.22 & 2.92 & 3.89 & $<0.001$ & $2.48-15.62$ & 1.83 & 6 \\
\hline \multicolumn{8}{|c|}{ Largest tumor diameter $(\mathrm{cm})$} \\
\hline$\leq 4$ & 1 & - & - & - & - & - & 0 \\
\hline $4-6$ & 2.17 & 0.83 & 2.03 & 0.043 & $1.01-4.57$ & 0.77 & 2 \\
\hline $6-8$ & 3.09 & 1.32 & 2.64 & 0.008 & $1.34-7.16$ & 1.13 & 3 \\
\hline$>8$ & 12.82 & 4.43 & 7.39 & $<0.001$ & $6.52-25.24$ & 2.55 & 13 \\
\hline \multicolumn{8}{|c|}{ Nodules number } \\
\hline Single & 1 & - & - & - & - & - & 0 \\
\hline Multiple & 2.54 & 0.71 & 3.36 & 0.001 & $1.48-4.38$ & 0.93 & 3 \\
\hline
\end{tabular}

0-5: low risk, 6-8 medium risk, $>8$ high risk
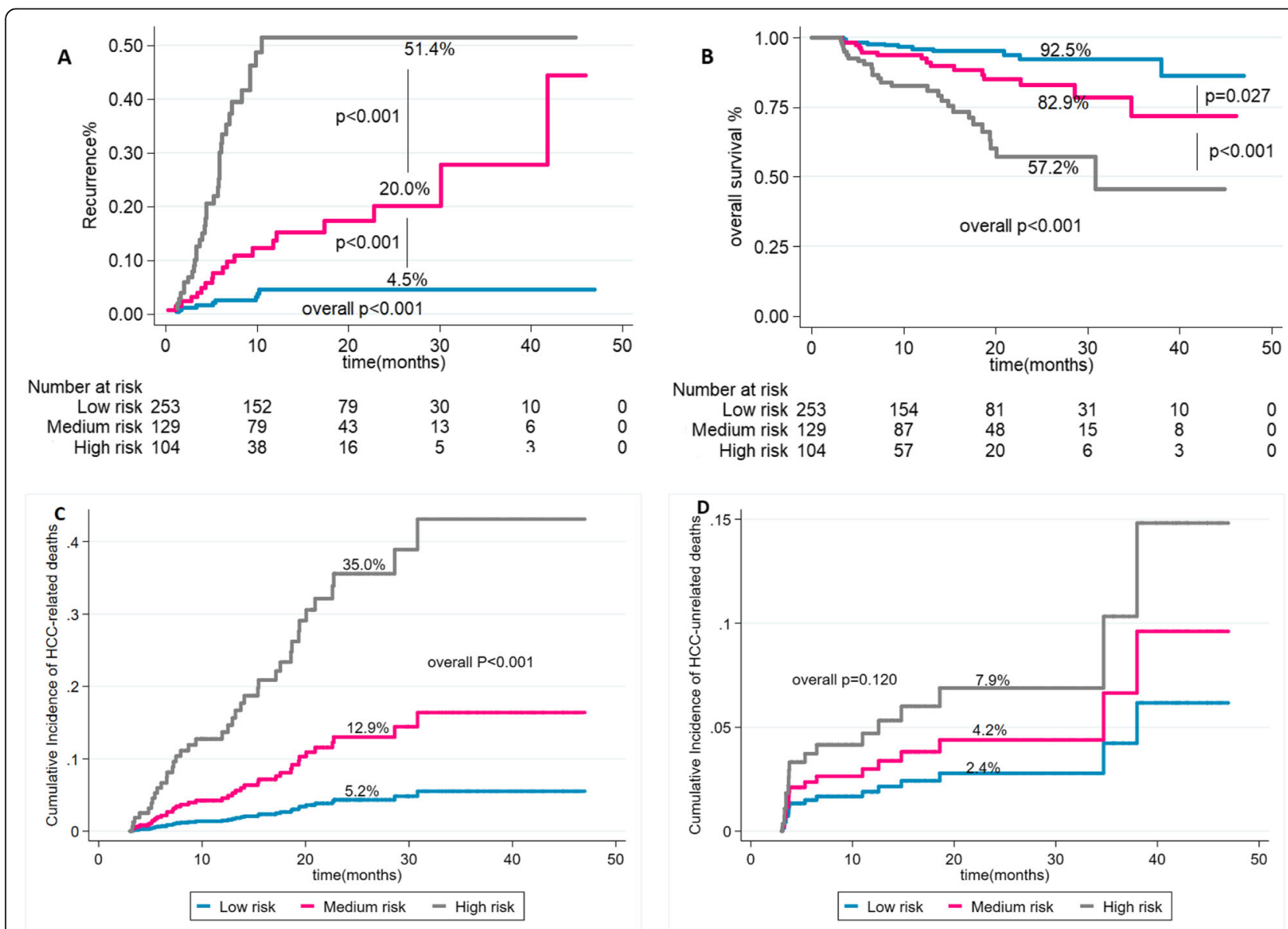

Fig. 2 In the training cohort and according to the 5-8 model, the 2-year recurrence rates (a) and overall survival rates(b). The cumulative incidence of HCC-related deaths (c) and HCC-unrelated deaths(d) as assessed by the competing risk analysis 
Table 4 Accuracy of the 5-8 model for predicting the risk of HCC early recurrence in the training and validation cohort compared with Milan, Hangzhou, and AFP model

\begin{tabular}{llllll}
\hline & \multicolumn{1}{l}{ Training cohort } & & \multicolumn{2}{l}{ Validation cohort } \\
\cline { 2 - 3 } & Harrell's C $(95 \%$ Cl) & Somer's D (95\% Cl) & & Harrell's C (95\% Cl) & Somer's D (95\% Cl) \\
\hline $5-8$ model & $0.79(0.73-0.86)$ & $0.59(0.40-0.72)$ & $0.74(0.66-0.82)$ & $0.49(0.32-0.74)$ \\
Milan & $0.72(0.67-0.76)$ & $0.43(0.35-0.58)$ & $0.65(0.59-0.71)$ & $0.30(0.17-0.45)$ \\
Hangzhou & $0.72(0.65-0.78)$ & $0.43(0.31-0.61)$ & $0.61(0.54-0.69)$ & $0.23(0.07-0.40)$ \\
AFP model & $0.72(0.66-0.77)$ & $0.43(0.33-0.60)$ & $0.68(0.60-0.75)$ & $0.35(0.20-0.53)$ \\
\hline
\end{tabular}

patients who were within AFP model (62\%), the risk of 2-year HCC recurrence was $4.0 \%$ (95\% CI: $0.02-0.09$ ) in the low-risk group, $19.6 \%(95 \%$ CI: $0.09-0.43)$ in medium- risk group ( $p=0.006)$ (Fig. $4 \mathrm{~b})$. While for 185 patients who are exceeding AFP model (38.1\%), the risk of 2-year HCC recurrence was 9.8\% (95\% CI: 0.02-0.39),
20.8\% (95\% CI: $0.11-0.41)$, and $51.4 \%(95 \%$ CI: $0.36-$ 0.73 ), respectively (overall $p=0.006$ ) (Fig. 4c).

Validation of the 5-8 model

As mentioned above, there were no significant differences in baseline characteristics among the training and
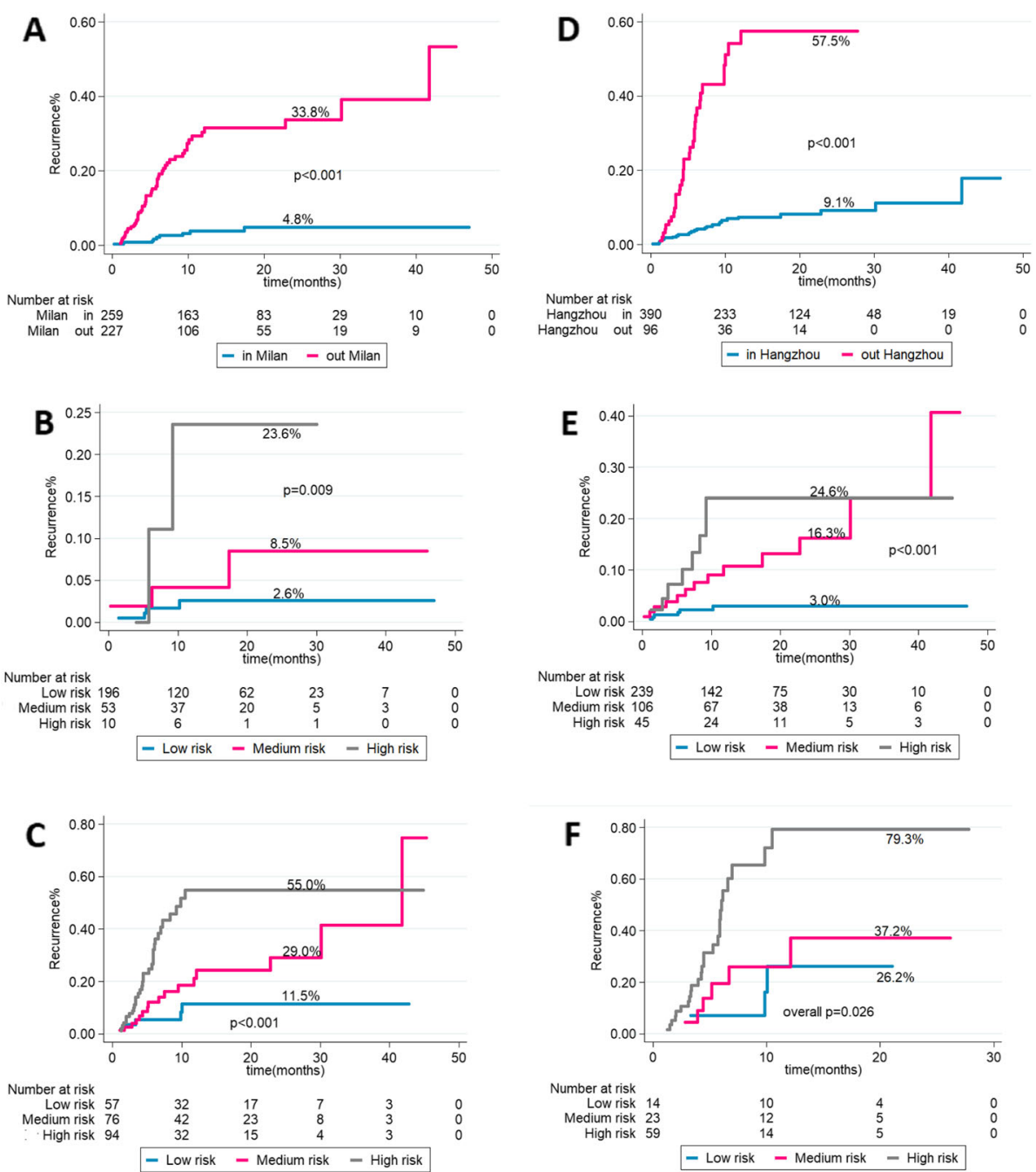

Fig. 3 In the training cohort, 2-year recurrence rates according to Milan criteria (a), according to 5-8 model in patients fulfilling Milan criteria (b) and in patients exceeding Milan criteria (c). Two-year recurrence rates according to Hangzhou criteria (d), according to the 5-8 model in patients fulfilling Hangzhou criteria (e) and in patients exceeding Hangzhou criteria (f) 

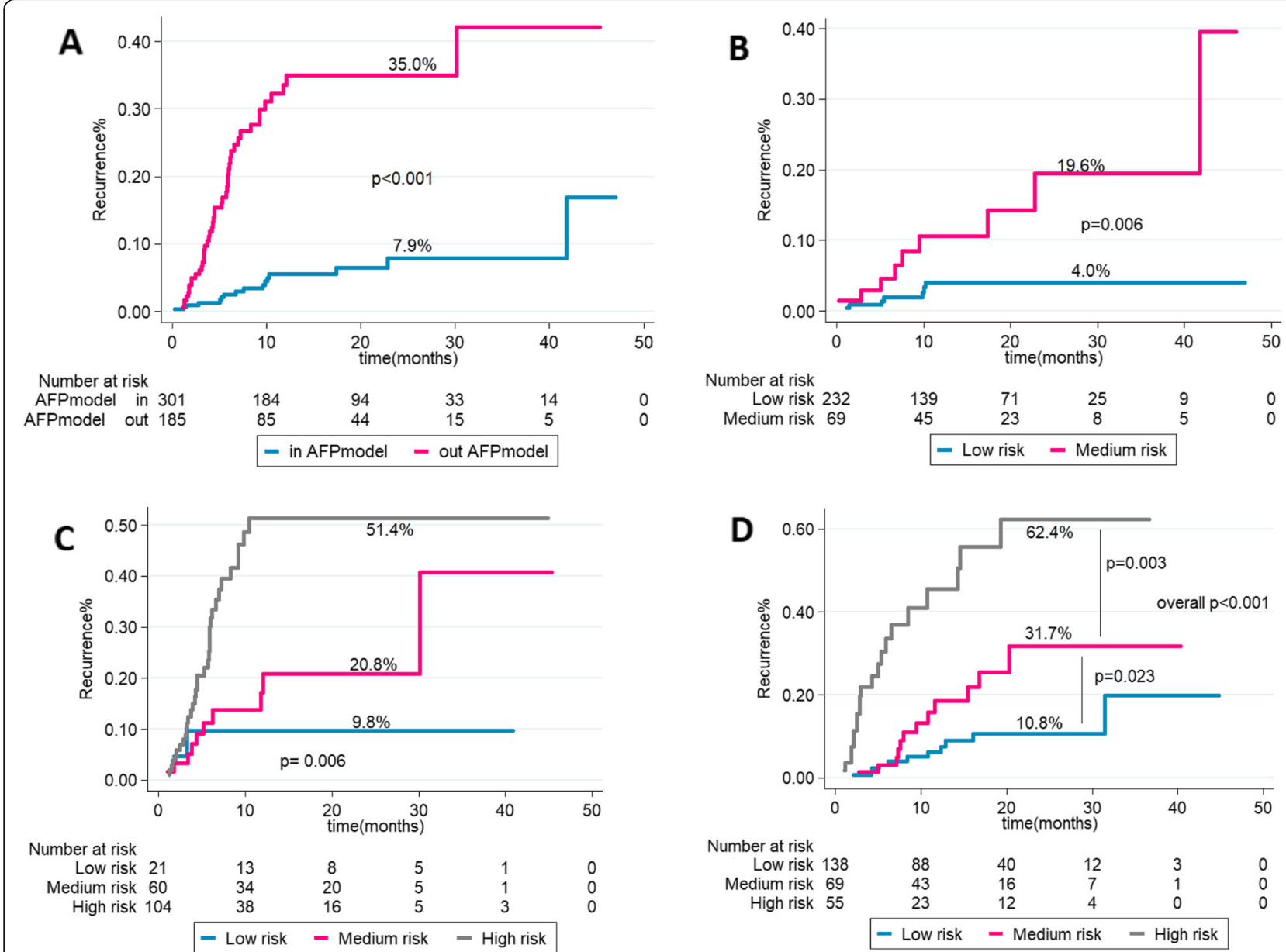

Fig. 4 In the training cohort, the 2-year recurrence rates according to AFP model (a), according to the 5-8 model for patients within the AFP model (b) and in patients outside AFP model (c). In the validation cohort, the 2-year recurrence rates (d) according to the 5-8 model

validation cohort. In the validation cohort, the median post-LT follow-up was 416.5 days [IQR:205-672]. The 5-8 model could also accurately stratify patients into low, medium and high-risk prognostic subgroups with 2-year HCC recurrence rates of 10.8\% (95\% CI: $0.06-$ $0.21), 31.7 \%$ (95\% CI: $0.17-0.59)$ and $62.4 \%$ (95\% CI: $0.39-1.00$ ) respectively, (overall log-rank $p<0.001$ ) (Fig. 4d). The 5-8 score also achieved a good performance in post-LT recurrence risk prediction in the validation cohort according to the visual assessment of the resulting KM curves and the Harrell's C and Somers' D of 74\%(95\% CI:0.66-0.82) and 49\%(95\% CI:0.32-0.74) compared with 65\%(95\% CI:0.59-0.71) and 30\%(95\% CI: 0.17$0.45)$ for Milan criteria and $61 \%(95 \%$ CI:0.54-0.69), 23\%(95\% CI:0.07-0.40) for Hangzhou criteria (Table 4).

\section{Discussion}

Recurrence of HCC after LT remains a major obstacle and associated with an unfavorable prognosis [20]. Several independent risk factors for post-LT HCC recurrence have been identified including number and size of tumors on preoperative imaging studies $[11,12,21]$ and pre-LT serum levels of AFP at different cut-off points: $10,200,1000 \mathrm{ng} / \mathrm{mL}$ [22-25]. However, lack of agreement about accurate, reliable and robust validated tool especially for prediction of early recurrence of HCC in HBV predominant population, make appropriate risk stratification and doctor-patient communication challenging.

In the present study, 786 patients with HCC diagnosed by imaging who underwent deceased donor LT from centers distributed throughout the whole China were involved and the 5-8 score was developed and validated. Our predictive model is a simple and reliable tool that showed excellent stratification of HCC patients into three risk subgroups; low-, medium- and high-risk with predicted 2-year recurrence ranging from $5 \%$ in low-risk to $20.0 \%$ in the medium risk and $51 \%$ in the high-risk category. For the 253 patients $(52.1 \%)$ in the low-risk group, the 2 - year OS was $93 \%$, significantly superior to the 83 and $57 \%$ of the medium- and high-risk groups.

Similar to our study, previous risk models [16, 26-28] have attempted to employ pre-LT AFP, largest tumor 
diameter and the number of nodules to predict the postLT HCC recurrence, but the characteristics of their study population (western participants with predominantly HCV) was different. Our model is mainly based on the data of patients with $\mathrm{HCC}$ occurring in patients with HBV infection (96\%) which is the most common cause of HCC in China. The high predictive accuracy of our preoperative model [Harrell's C was $79 \%(95 \%$ CI: $0.73-$ $0.86)$ and $74 \%(95 \%$ CI:0.66-0.82), respectively, in the training and validation cohorts] stems from the utilization of three preoperative factors with more accurate cut-off points that were strongly associated with $\mathrm{HCC}$ recurrence: pre-LT AFP $(10-200, \quad 201-1000$, and $>1000 \mathrm{ng} / \mathrm{mL})$, largest tumor diameter $(4-6,6.1-8,>8 \mathrm{~cm})$, and the number of nodules (single vs multiple). Unlike previous studies, our model end-point was recurrence at 2-year which is the discriminative cut-off value for early and late recurrence of HCC, however, the precise cut-off may require genetic/genomic analyses recurrence. Early recurrence results from preexisting tumor cells while late recurrence or de novo tumor mainly arising due to new malignant clones [29-31]. Using the cut-off values in our study was a clinical decision based on the expert's assessment and they achieved the lowest AIC values so better fit the model.

Furthermore, one of the main advantage of our score over previous mentioned models is its ability to discriminate three subgroups of HCC recurrence(i.e low,medium, high), while other models only classify HCC patients into two risk subgroups of HCC recurrence, high and low risk. The categorization defining only two subgroups at risk is not valuable, or at least less practical. For example, tumour recurrence risk divided into low $(<8 \%)$ and high $(>50 \%)$ will produces a big 'grey zone' through $8-50 \%$ of mediumrisk individuals [32]. Our score solved this drawback by stratify the individuals at risk into three subgroups of risk of recurrence, low,medium and high. This can be translated clinically into an excellence of individuals selections for LT, a more reasonable organs allocation taking into account the donor offer, besides, and the opportunity to stratify risk for the development of upcoming adjuvant treatments [33].

One of the most interesting results comes when we compared our model with the conventional selection criteria by which our model can stratify patients within and outside the Milan and Hangzhou criteria. For instance, the subgroup of patients $(25 \%)$ who were recognized as high risk of recurrence by Milan (out Milan), they carry a low risk according to our score with 2-year recurrence probability of $12 \%$. While the subgroup of patients(4\%) who were recognized as low risk of recurrence by Milan (in Milan), they carry a high risk according to our score with 2 -year recurrence probability of $24 \%$. Similarly, the subgroup of patients (12\%) who were recognized as low risk of recurrence by Hangzhou (in Hangzhou), they carry a high risk according to our score with 2-year recurrence probability of $25 \%$. The addition of our model to the conventional selection criteria may, therefore, allow us to capture accurately the patients with a higher risk of recurrence who were traditionally considered as the lowest risk group. External validation of this preoperative adjuvant model is mandatory to help in more accurate selection of HCC patients for LT and to avoid the high post-LT recurrence probabilities.

Moreover, one of the advantages of our model is when it was compared to the AFP model, about (11 and 32\%) of patients recognized as high risk of recurrence by AFP model, but they had a low and medium risk according to our score with 2-year recurrence probability of $10,21 \%$ respectively. Also, about $23 \%$ of the patients were defined as a low risk of recurrence by the AFP model, but a medium risk of recurrence was revealed by our score with 2-year recurrence probability of $20 \%$. Another advantage of our model is the outcome of the competing risk analysis, the rates of HCC-unrelated death were similar $(p=0.120)$ and the rates of HCC-related death which mainly due to $\mathrm{HCC}$ recurrence were significantly different $(p<0.001)$, indicating that the $5-8$ model is a powerful tool to pick up the HCC recurrence but not other causes of deaths and this explains the differences in survival rates according to the 5-8 model.

At this point, we need a further explanation for the clinical application of our model. The patients presented preoperatively with a single nodule, diameter of $\leq 4 \mathrm{~cm}$ and serum AFP of $\leq 10 \mathrm{ng} / \mathrm{mL}$ will fall in the low-risk group by getting the $5-8$ score of 0 and this will effectively exclude the probability of post-LT HCC recurrence, so these patients can go directly for LT. However,The patients with largest nodule diameter of $>8 \mathrm{~cm}$ and serum AFP of $>1000 \mathrm{ng} / \mathrm{mL}$ will be categorized in the high-risk category (Table 3), so LT should be excluded or neoadjuvant therapy and close surveillance until the tumor biology and morphology could be brought down to a safer level. However, patients who belong to the medium HCC recurrence risk category (e.g. single nodule, diameter of $8 \mathrm{~cm}$ and serum AFP of $700 \mathrm{ng} / \mathrm{mL}$ ), a careful selection for LT based on a personalized assessment and pre-LT downstaging therapy should be considered then early administration of mTOR inhibitor postoperatively are strongly recommended [34].

Our study has some limitations. First, its retrospective nature so we designed a prospective study to confirm the clinical utility of our model. Second, the characteristics of our study population (predominantly HBV infected male Chinese patients) so external validation in $\mathrm{HCV}$ predominant, non-Chinese populations is required to confirm the reliability of the 5-8 model. Third, the patients with vascular invasion were not excluded from our study. Fourth,some parameters, such as 
microvascular invasion and data regarding response to pre-LT neoadjuvant therapies are incomplete in the CLTR database and this precludes us from comparing our model with other prominent risk scores. Lastly, although the follow-up time was enough to pick up the events, it is relatively short in comparison with other studies.

\section{Conclusions}

In conclusion, a preoperative predictive risk score for early recurrence of HCC after LT was constructed using 3 variables (pre-LT AFP, largest tumor diameter, and the number of nodules). Our model accurately predicts early recurrence of $\mathrm{HCC}$ at an individual level in patients with HBV-cirrhosis. It could effectively classify HCC patients into subgroups with a low, medium and high risk of recurrence and can potentially be used to guide therapeutic decisions and facilitate risk communication between surgeons and patients. We believe that our model can play a complementary role in the selection of HCC patients for LT and prediction of early recurrence alongside the conventional selection criteria. Prospective validation of our model will be an important step to verify its clinical utility.

\section{Abbreviations}

AFP: Alpha-fetoprotein; BMI: Body mass index; Cl: Confidence interval; CLTR: China Liver Transplant Registry; CVA: Cerebrovascular accident; DBCD: Donation after brain death followed by circulatory death; DBD: Donation after brainstem death; DCD: Donation after circulatory death; HBV: Hepatitis B virus; HCC: Hepatocellular carcinoma; HR: Hazard ratio; IQR: Interquartile range; LT: Liver transplantation; MELD: Model for End-Stage Liver Disease

\section{Acknowledgments}

We would like to thank China Liver Transplantation Registry (CLTR) for their help in the data acquisition and extraction. We also extend our thanks to the transplant centers from Mainland China for their major contribution to the CLTR database.

\section{Authors' contributions}

$A A, X X, S Z$ designed the study, $H X, L Z, X X$, and $S Z$ give the administrative support, $X W, L L, Z S$, and $H G$ provide the study materials and collect the data, $A A$ and $X X$ analyse the data and interpret the results. All authors read and approved the final manuscript.

\section{Funding}

This work supported by National S\&T Major Project (Grant number:2017ZX10203205); Medical Science and Technology Project of Zhejiang Province (Grant number:2016146968) and China Postdoctoral Science Foundation (Grant number:2017 M612014). The above funding bodies had no further role in the study design; in the collection, analysis and interpretation of data; in the writing of the article or in the decision to submit the article for publication.

\section{Availability of data and materials}

The data that support the findings of this study are available from the China Liver Transplant Registry but restrictions apply to the availability of these data, which were used under license for the current study, and so are not publicly available. Data are however available from the authors upon reasonable request and with permission of the China Liver Transplant Registry.

\section{Ethics approval and consent to participate}

This study approved by the Scientific Committee of the China Liver Transplant Registry (http://www.cltr.org/) which is in accordance with ethical guidelines of Helsinki Declaration 1975, as revised in 2013. Every participant had received a written informed consent before being included in this study.

\section{Consent for publication}

Not applicable.

\section{Competing interests}

The authors declare that they have no competing interests.

\section{Author details}

'Department of Hepatobiliary and Pancreatic Surgery, First Affiliated Hospital, Zhejiang University School of Medicine; Institution of Organ Transplantation, Zhejiang University, Hangzhou 310003, China. ${ }^{2}$ NHFPC Key Laboratory of Combined Multi-organ Transplantation, Hangzhou 310003, Zhejiang Province, China. ${ }^{3}$ China Liver Transplant Registry, Hangzhou, China.

Received: 8 August 2019 Accepted: 7 November 2019

Published online: 21 November 2019

\section{References}

1. Forner A, Reig M, Bruix J. Hepatocellular carcinoma. Lancet (London, England). 2018:391(10127):1301-14.

2. Wong MCS, Huang JLW, George J, Huang J, Leung C, Eslam M, et al. The changing epidemiology of liver diseases in the Asia-Pacific region. Nat Rev Gastroenterol Hepatol. 2019;16(1):57-73.

3. Sapisochin G, Bruix J. Liver transplantation for hepatocellular carcinoma: outcomes and novel surgical approaches. Nat Rev Gastroenterol Hepatol. 2017;14(4):203-17.

4. Mazzaferro V, Regalia E, Doci R, Andreola S, Pulvirenti A, Bozzetti F, et al. Liver transplantation for the treatment of small hepatocellular carcinomas in patients with cirrhosis. N Engl J Med. 1996;334(11):693-9.

5. Zheng SS, Xu X, Wu J, Chen J, Wang WL, Zhang M, et al. Liver transplantation for hepatocellular carcinoma: Hangzhou experiences. Transplantation. 2008:85(12):1726-32.

6. Sharma P, Welch K, Hussain H, Pelletier SJ, Fontana RJ, Marrero J, et al. Incidence and risk factors of hepatocellular carcinoma recurrence after liver transplantation in the MELD era. Dig Dis Sci. 2012;57(3):806-12.

7. Xu X, Lu D, Ling Q, Wei X, Wu J, Zhou L, et al. Liver transplantation for hepatocellular carcinoma beyond the Milan criteria. Gut. 2016;65(6):1035-41.

8. Berry K, loannou GN. Serum alpha-fetoprotein level independently predicts posttransplant survival in patients with hepatocellular carcinoma. Liver Transplant. 2013:19(6):634-45.

9. Lee JH, Cho Y, Kim HY, Cho EJ, Lee DH, Yu SJ, et al. Serum tumor markers provide refined prognostication in selecting liver transplantation candidate for hepatocellular carcinoma patients beyond the Milan criteria. Ann Surg. 2016;263(5):842-50.

10. Xiao WK, Chen D, Li SQ, Fu SJ, Peng BG, Liang LJ. Prognostic significance of neutrophil-lymphocyte ratio in hepatocellular carcinoma: a meta-analysis. BMC Cancer. 2014;14:117.

11. Mazzaferro V, Llovet JM, Miceli R, Bhoori S, Schiavo M, Mariani L, et al. Predicting survival after liver transplantation in patients with hepatocellular carcinoma beyond the Milan criteria: a retrospective, exploratory analysis. Lancet Oncol. 2009;10(1):35-43.

12. Germani G, Gurusamy K, Garcovich M, Toso C, Fede G, Hemming A, et al. Which matters most: number of tumors, size of the largest tumor, or total tumor volume? Liver Transplant. 2011;17(Suppl 2):S58-66.

13. Collins GS, Reitsma JB, Altman DG, Moons KG. Transparent reporting of a multivariable prediction model for individual prognosis or diagnosis (TRIPOD): the TRIPOD statement. BMJ (Clinical research ed). 2015;350:g7594.

14. Marrero JA, Kulik LM, Sirlin CB, Zhu AX, Finn RS, Abecassis MM, et al. Diagnosis, staging, and Management of Hepatocellular Carcinoma: 2018 practice guidance by the American Association for the Study of Liver Diseases. Hepatology (Baltimore, Md). 2018;68(2):723-50.

15. Edmondson HA, Steiner PE. Primary carcinoma of the liver: a study of 100 cases among 48,900 necropsies. Cancer. 1954;7(3):462-503.

16. Duvoux C, Roudot-Thoraval F, Decaens T, Pessione F, Badran H, Piardi T, et al. Liver transplantation for hepatocellular carcinoma: a model including 
alpha-fetoprotein improves the performance of Milan criteria. Gastroenterology. 2012;143(4):986-94 e3; quiz e14-5.

17. EASL clinical practice guidelines: management of hepatocellular carcinoma. J Hepatol. 2018;69(1):182-236.

18. Newson RB. Comparing the predictive powers of survival models using Harrell's C or Somers' D. Stata J. 2010;10(3):339-58.

19. Fine JP, Gray RJ. A proportional hazards model for the subdistribution of a competing risk. J Am Stat Assoc. 1999;94(446):496-509.

20. Roayaie S, Schwartz JD, Sung MW, Emre SH, Miller CM, Gondolesi GE, et al. Recurrence of hepatocellular carcinoma after liver transplant: patterns and prognosis. Liver Transplant. 2004;10(4):534-40.

21. Marelli L, Grasso A, Pleguezuelo M, Martines H, Stigliano R, Dhillon AP, et al. Tumour size and differentiation in predicting recurrence of hepatocellular carcinoma after liver transplantation: external validation of a new prognostic score. Ann Surg Oncol. 2008;15(12):3503-11.

22. Agopian VG, Harlander-Locke MP, Markovic D, Zarrinpar A, Kaldas FM, Cheng EY, et al. Evaluation of patients with hepatocellular carcinomas that do not produce alpha-fetoprotein. JAMA Surg. 2017;152(1):55-64.

23. Lee S, Hyuck David Kwon C, Man Kim J, Joh JW, Woon Paik S, Kim BW, et al. Time of hepatocellular carcinoma recurrence after liver resection and alphafetoprotein are important prognostic factors for salvage liver transplantation. Liver Transplant. 2014;20(9):1057-63.

24. Hong G, Suh KS, Suh SW, Yoo T, Kim H, Park MS, et al. Alpha-fetoprotein and (18) F-FDG positron emission tomography predict tumor recurrence better than Milan criteria in living donor liver transplantation. J Hepatol. 2016:64(4):852-9.

25. Hameed B, Mehta N, Sapisochin G, Roberts JP, Yao FY. Alpha-fetoprotein level $>1000 \mathrm{ng} / \mathrm{mL}$ as an exclusion criterion for liver transplantation in patients with hepatocellular carcinoma meeting the Milan criteria. Liver Transplant. 2014;20(8):945-51.

26. Agopian VG, Harlander-Locke M, Zarrinpar A, Kaldas FM, Farmer DG, Yersiz $\mathrm{H}$, et al. A novel prognostic nomogram accurately predicts hepatocellular carcinoma recurrence after liver transplantation: analysis of 865 consecutive liver transplant recipients. J Am Coll Surg. 2015;220(4):416-27.

27. Mehta N, Heimbach J, Harnois DM, Sapisochin G, Dodge JL, Lee D, et al. Validation of a risk estimation of tumor recurrence after transplant (RETREAT) score for hepatocellular carcinoma recurrence after liver transplant. JAMA Oncol. 2017;3(4):493-500.

28. Halazun K, Najjar M, Abdelmessih RM, Samstein B, Griesemer AD, Guarrera $\mathrm{JV}$, et al. Recurrence after liver transplantation for hepatocellular carcinoma: a new MORAL to the story. Ann Surg. 2017;265(3):557-64.

29. Imamura H, Matsuyama Y, Tanaka E, Ohkubo T, Hasegawa K, Miyagawa S, et al. Risk factors contributing to early and late phase intrahepatic recurrence of hepatocellular carcinoma after hepatectomy. J Hepatol. 2003; 38(2):200-7.

30. Vilarinho S, Calvisi DF. New advances in precision medicine for hepatocellular carcinoma recurrence prediction and treatment. Hepatology (Baltimore, Md). 2014;60(6):1812-4.

31. Kim JH, Sohn BH, Lee HS, Kim SB, Yoo JE, Park YY, et al. Genomic predictors for recurrence patterns of hepatocellular carcinoma: model derivation and validation. PLoS Med. 2014;11(12):e1001770.

32. Pinero F, Carrihlo FJ, Silva MO. Predictive models for recurrence risk of hepatocellular carcinoma after liver transplantation: still an unmet need. Liver Int. 2017;37(5):648-50.

33. Geissler EK, Schnitzbauer AA, Zulke C, Lamby PE, Proneth A, Duvoux C, et al. Sirolimus use in liver transplant recipients with hepatocellular carcinoma: a randomized, multicenter, open-label phase 3 trial. Transplantation. 2016; 100(1):116-25.

34. Duvoux C, Toso C. mTOR inhibitor therapy: does it prevent HCC recurrence after liver transplantation? Transplant Rev (Orlando). 2015;29(3):168-74.

\section{Publisher's Note}

Springer Nature remains neutral with regard to jurisdictional claims in published maps and institutional affiliations.

\section{Ready to submit your research? Choose BMC and benefit from:}

- fast, convenient online submission

- thorough peer review by experienced researchers in your field

- rapid publication on acceptance

- support for research data, including large and complex data types

- gold Open Access which fosters wider collaboration and increased citations

- maximum visibility for your research: over $100 \mathrm{M}$ website views per year

At BMC, research is always in progress.

Learn more biomedcentral.com/submissions 